\title{
Measuring Individual Disaster Recovery: A Socioecological Framework
}

\author{
David M. Abramson, PhD, MPH; Tasha Stehling-Ariza, MPH; Yoon Soo Park, MS;
}

Lauren Walsh, MPH; Derrin Culp, MCRP, MIA

\section{ABSTRACT}

Background: Disaster recovery is a complex phenomenon. Too often, recovery is measured in singular fashion, such as quantifying rebuilt infrastructure or lifelines, without taking in to account the affected population's individual and community recovery. A comprehensive framework is needed that encompasses a much broader and far-reaching construct with multiple underlying dimensions and numerous causal pathways; without the consideration of a comprehensive framework that investigates relationships between these factors, an accurate measurement of recovery may not be valid. This study proposes a model that encapsulates these ideas into a single framework, the Socio-Ecological Model of Recovery.

Methods: Using confirmatory factor analysis, an operational measure of recovery was developed and validated using the five measures of housing stability, economic stability, physical health, mental health, and social role adaptation. The data were drawn from a sample of displaced households following Hurricane Katrina. Measures of psychological strength, risk, disaster exposure, neighborhood contextual effects, and formal and informal help were modeled to examine their direct and indirect effects on recovery using a structural equation model.

Findings: All five elements of the recovery measure were positively correlated with a latent measure of recovery, although mental health and social role adaptation displayed the strongest associations. An individual's psychological strength had the greatest association with positive recovery, followed by having a household income greater than $\$ 20000$ and having informal social support. Those factors most strongly associated with an absence of recovery included the time displaced since the hurricane, being disabled, and living in a community with substantial social disorder.

Discussion: The socio-ecological framework provides a robust means for measuring recovery, and for testing those factors associated with the presence or absence of recovery.

(Disaster Med Public Health Preparedness. 2010;4:S46-S54)

Key Words: recovery, socioecological model, mediators, structural equation model

I $\mathrm{n}$ the aftermath of a disaster, measuring recovery is essential for planners and policy makers interested in restoring economic and social activity. Communitylevel measures of the built environment such as houses rebuilt, roads repaired, and critical infrastructure restored can capture such a rebound. Economic measures that chart an area's return to financial health serve a similar purpose. Often left unmeasured, however, is how well the affected populations are recovering. Although current research indicates community- and individual-level recovery are not one and the same, a robust measure of long-term individual recovery has yet to be developed.

Much theoretical and empirical work has been advanced regarding human consequences of disasters, but the basis for understanding how individuals recover and what exactly constitutes recovery is still evolving. There is a tension between these 2 questions: how formulates recovery as a process, whereas what considers recovery as an outcome. Social theorists have advanced vulnerability theories, for example, to explain why certain subpopulations are more susceptible to the disaster stress- ors. ${ }^{1}$ Stress theories explain the mechanisms by which individuals and households experience stress in a postdisaster setting. ${ }^{2}$ Social cognition theory offers an explanation as to how individual traits and characteristics, such as coping self-efficacy, can buffer a disaster's micro- and macrostresses. ${ }^{3}$ Finally, recent work on community resiliency has begun to elucidate the role of community-level adaptive capacities in individual recovery. ${ }^{4}$ These distinct frameworks suggest that there are multiple interacting pathways or processes that lead to full or partial restoration of an individual's circumstances after a disaster. Achieving a state of recovery, then, could involve a combination of measures-relief of stressors, mitigation of vulnerability, enhancement of individual buffering capacities, and increased adaptive capacities — at a minimum. Indeed, many researchers would agree that one cannot meaningfully investigate the concept of recovery outside the context of complex social, economic, and political systems. ${ }^{5,6}$

A measure of recovery needs to capture this complexity. Previous studies have typically focused on 1-dimen- 
sional measures of recovery (eg, mental health, employment, housing stability) rather than incorporating multiple indicators of recovery into a single measure. This study proposes and validates an operational measure of recovery that incorporates multiple dimensions of an individual's recovery after a disaster. These dimensions include stable housing, good mental and physical health, stable economic circumstances, and positive social role adaptation. Following the development of a comprehensive outcome measure, we use a socioecological framework to investigate factors that mediate and moderate the causal pathways leading to recovery.

\section{SOCIOECOLOGICAL MODEL OF RECOVERY}

Socioecological models can help the researcher hypothesize and articulate complex interactions among interpersonal, organizational, community, and social systems and their subsequent effects on health. ${ }^{7}$ The models help conceptualize factors involved in the causal process and illustrate which variables are incorporated as a part of the outcome measure itself. Use of such a framework allows for the integration of multiple causal processes at various systemic levels to better understand complex pathways to a multidimensional outcome. In the context of a long-term individual recovery model, a socioecological framework can help researchers visualize potential relations between individual characteristics and exposures and the recovery outcome. Socioecological models, furthermore, allow researchers to incorporate the many indicators of recovery established in the literature in a more encompassing recovery outcome measure. To date, few disaster researchers have operationalized this type of framework in this context. In their 2003 and 2005 analyses of life recovery among survivors of the 1995 Kobe, Japan, earthquake. Tatsuki, Koshiyama, and Hayashi used a socioecological approach to investigate the life recovery process and individual recovery as an outcome. ${ }^{8-10}$ They use the term life recovery to encompass the many social aspects of recovery noted earlier and to distinguish it from infrastructure recovery that focuses on bricks and mortar. Although the socioecological framework has been used in other public health research domains, Tatsuki, Koshiyama, and Hayashi are, to our knowledge, unique in applying such a framework within recovery research.

As described previously, a multitude of factors may be included in socioecological models. The research literature consistently has identified improvements in or attainment of 5 dimensions of individual circumstances as indicators of recovery: housing stability, stable economic resources, good mental health, good physical health, and positive social role adaptation. Housing stability and consistent economic resources are among the most widely accepted indices of postdisaster recovery. Researchers have identified stable housing as an underlying foundation for recovery and have concluded that "delays in reestablishing housing all too often delay all other dimensions of recovery." ${ }^{11,12}$ Economic stability parallels housing stability in its role within long-term individual recovery. A number of studies have demonstrated that access to economic resources among disaster victims serves to buffer or mitigate the effects of the disaster., ${ }^{4,13-15}$
Good mental and physical health also have been used widely as indicators of individual recovery. In terms of mental health, the literature is still rife with disagreements about whether disasters cause clinical disorders, which disorders, and their severity and duration. ${ }^{16}$ It is largely agreed upon, however, that recovery implies, among other outcomes, a return to some kind of routine, normalcy, or stability, even if at a lower absolute level of satisfaction or welfare than before the event. Such routine or normalcy restores the ability to feel both safe and contented with one's life circumstances. ${ }^{4,17}$ In terms of physical health, separation from familiar objects, surroundings, and people due to involuntary relocation has been shown to increase helplessness and despair and lead to increased physical impairments. ${ }^{18}$ Social role adaptation is a fifth dimension of individual circumstances that has been identified as indicative of individual recovery. As described above, Tatsuki et al have articulated a life recovery model that substantiates the importance of adaptive social roles and communal identities in recovery processes. ${ }^{8-10}$ Mental health researchers also demonstrate that the more successful recovery policies and programmatic interventions are those that support social role adaptation and its connection to place. ${ }^{19}$ Community engagement, indigenous support systems, and locally acceptable recovery activities (including mutual assistance, charity, pioneering entrepreneurship and actions such as the reopening of a church or school) reinforce families' sense of safety and security and are believed to contribute to better overall recovery. ${ }^{20-22}$

The present analysis used a socioecological framework to develop a durable measure of individual recovery and explored how communal, intrapersonal, and interpersonal factors are associated with recovery. The objectives of this analysis were to develop an operational measure of individual recovery that incorporates mental health, housing, economic, and social domains and to assess how mediators and moderators influence recovery.

\section{METHODS}

\section{Data}

The empirical data used for this study come from the Gulf Coast Child \& Family Health (GCAFH) project, which enrolled 1079 Louisiana and Mississippi households displaced or heavily affected by Hurricane Katrina. Respondents were recruited 6 to 12 months after the hurricane using randomly sampled Federal Emergency Management Agency-subsidized housing sites and census blocks identified as moderately to extensively damaged using Federal Emergency Management Agency assessments. For the purposes of this analysis, cross-sectional data collected from the 844 respondents interviewed at the fourth wave of data collection ( $41 / 2$ years postdisaster) were used. A bias analysis showed that there were no significant differences between the baseline cohort and those interviewed at wave 4. A detailed account of the sampling framework and a description of the cohort at recruitment can be found in a previous issue of Disaster Medicine and Public Health Preparedness. ${ }^{23}$ 


\section{Developing a Psychometrically Valid Measure of Recovery}

In disciplines including the medical, psychological, educational, and social fields, variables that describe substantive theory are classified into 2 types: observed (manifest) and unobserved (latent). Examples of the former are variables that can be measured directly such as income, number of siblings, or weight; in their basic forms, all directly collected data are observed measurements. There are instances, however, when it becomes necessary to study unobserved variables (eg, intelligence, depression, health conditions) that cannot be captured fully by a single observed variable. In practice, these latent variables can be measured using a combination of observed variables. For example, obesity is a latent variable that is assessed by accounting for observable measurements of body mass, waist, and hip indexes ${ }^{24}$; similarly, asthma is a latent variable represented by the manifest variables of hyperactive airways, lung function, and pulmonary inflammatory processes.

As described above, recovery encompasses many complexities and thus cannot be measured as a single observed variable. The present study examined recovery as a latent variable composed of 5 dimensions. The first dimension was stable housing, which was determined using a series of items designed to measure whether the respondent lived in permanent or stable housing and did not anticipate moving for at least 1 year. The second dimension was stable economic circumstances, which referred to sufficient economic resources to maintain a reasonable quality of life; this was measured by a stable household-level inflow of income through salary or wages from a full- or part-time job and/or Social Security benefits, and continued employment for 1 year. The third and fourth components of recovery were good physical and mental health. The constructs used were those defined in the Medical Outcomes Study Short-Form Version 2 (SF-12) instrument, which describes positive physical functioning, maintenance of good mental health, and positive psychological and emotional functioning. ${ }^{25,26}$ Finally, the fifth dimension was social role adaptation, which incorporated the family functioning scale used by Tatsuki and Hayashi to measure the strength of functional social ties within the household. ${ }^{10}$

The general methodological framework for this analysis uses the structural equation model (SEM) approach. ${ }^{27}$ In general, SEMs are regression models with both observed and latent variables and comprise measurement and structural components. The measurement component is used to create the latent variable (ie, the recovery measure from the 5 dimensions) and the structural component is used to model the various paths that construct the relations between the latent and observed variables. Figure 1 is a simplified diagram that illustrates both the measurement and structural components of the SEM used in this study. The final outcome of the model is "recovery," which is the lower-right circle of the diagram. There are 2 mediating factors- "neighborhood" and "help"- and 1 "exposure" variable. Neighborhood is another latent variable. It was measured using a scale comprising 14 items that were used to create 2 subfactors, social and physical disorder, and is a modified version of the neighborhood effects measure used by Tatsuki and Hayashi. ${ }^{10}$ Help was categorized into

\section{FIGURE 1}

\section{Socioecological model of postdisaster recovery.}

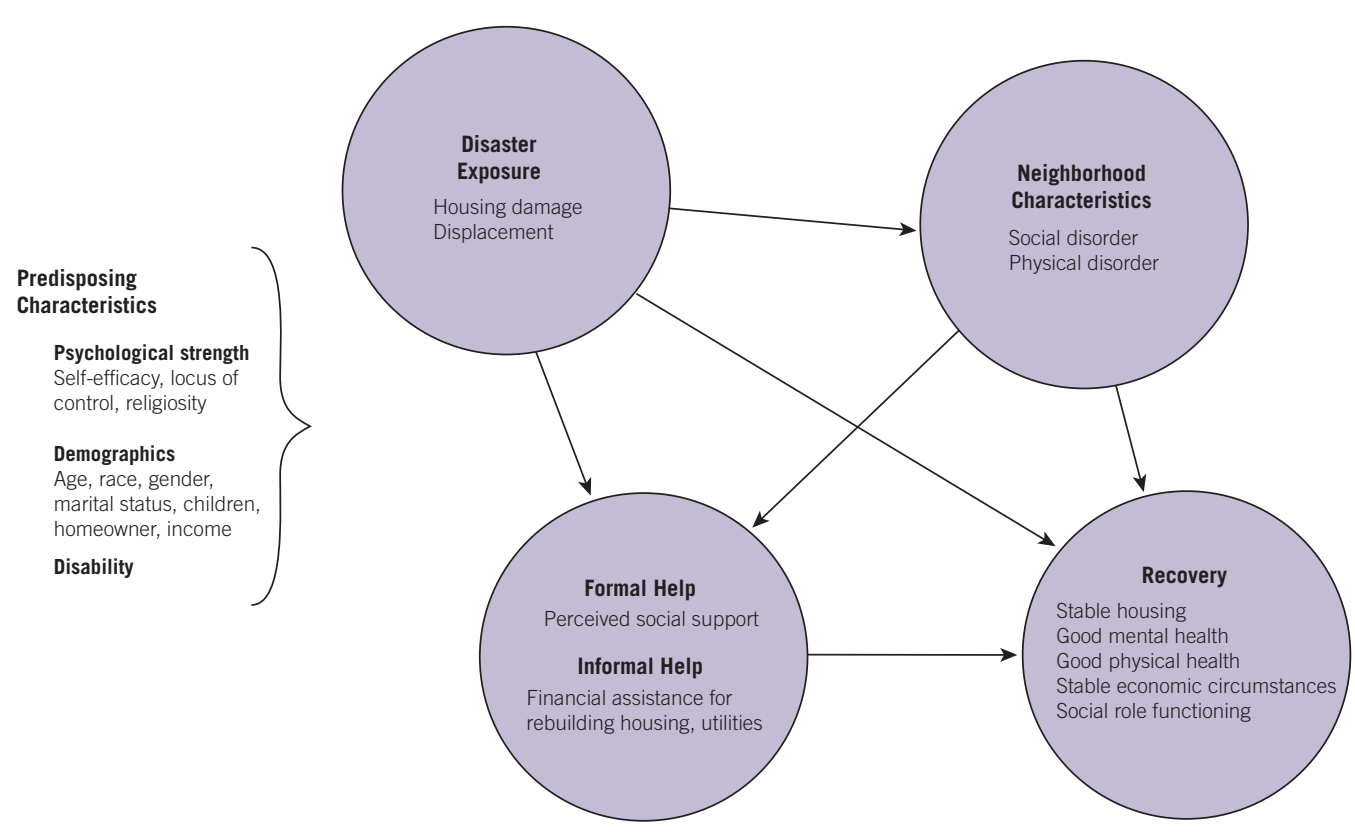


2 forms: (1) informal help, which included perceived and received social support, measured using a 5 -item scale on being able to rely on others for everyday favors, receiving health care, receiving economic support for medical emergency, receiving housing help, and having someone to talk to about family relationship troubles, and (2) formal help, which included having received relief services for immediate needs and help toward restoration and rehabilitation of housing. The exposure variable was observed using discrete measurements of years displaced and the extent of housing damage.

Two moderators were incorporated into the model: psychological strength and disability. The former was treated as a latent variable composed of 3 indicators: (1) self-efficacy (ie, the perceived capacity to adapt to stressors as measured by the general perceived self-efficacy scale from Schwarzer and Jerusalem ${ }^{28}$ ), (2) locus of control (ie, the purposive action and life consequences governed by an individual that indicated whether there were barriers in his or her life, outlook on success, and control of things happening in life), and (3) religiosity (ie, frequency of institutional attendance of religious services). Disability was a self-identified variable offered as an option when asked for occupational status in the GCAFH survey. Sociodemographic variables including age, race and ethnicity, sex, marital status, household composition, homeownership at the time of Katrina, and household income were included in the model as predisposing characteristics to control the effect of the outcome.

\section{Reliability and Validity of the Recovery Measure}

When developing a measure, both reliability and validity should be addressed. Reliability refers to consistency of measurements, whereas validity ensures the inclusion of relevant ideas used to develop the measure. As noted by Allen and Yen, satisfying validity infers reliability. ${ }^{29}$ There are various types of validity, how- ever, and among them, construct validity encompasses the broad definitions used by most researchers to quantify the extent to which an operationalization measures the concept it is supposed to measure. ${ }^{30} \mathrm{SEMs}$ provide an elegant method of achieving construct validity because measures of model fit can be used to establish validity. SEMs can also be simplified; when SEMs have measurement components and lack structural components for constructing the latent variable (ie, missing the regressional part of SEMs that models the relation between latent and observed variables), they become models of confirmatory factor analysis (CFA), another common tool for creating and validating measures. ${ }^{31}$

This study used 2 analytical steps that encompass both CFA and SEM. The first step involved developing and validating the latent variables used for this study, which included the operational measure of recovery from the 5 dimensions and factors that pertain to psychological strength and neighborhood effects (ie, treating social and physical disorder as separate but correlated factors). CFA was used to test whether the theorized variables were sufficient to create each latent factor. Indices of model fit from running CFA were examined to infer the satisfaction of construct validity of the hypothesized model. In the next step, recovery was tested for face validity, to test for association with a subjective measurement of recovery. This was done using an independent samples $t$ test. In the ensuing phase of the analytical plan, associations and pathways of factors leading to recovery were tested by combining the measurement (eg, CFA from the latent variables) and structural components (eg, multiple paths incorporating mediators and moderators) of the model into a simultaneous SEM. This final step completed the test for developing the socioecological model of recovery. Unlike traditional methods of path analysis, an SEM approach validates the proposed model with both its components and statistical error included under a single framework.

\section{FIGURE 2}

Factor loadings for the recovery measurement model.

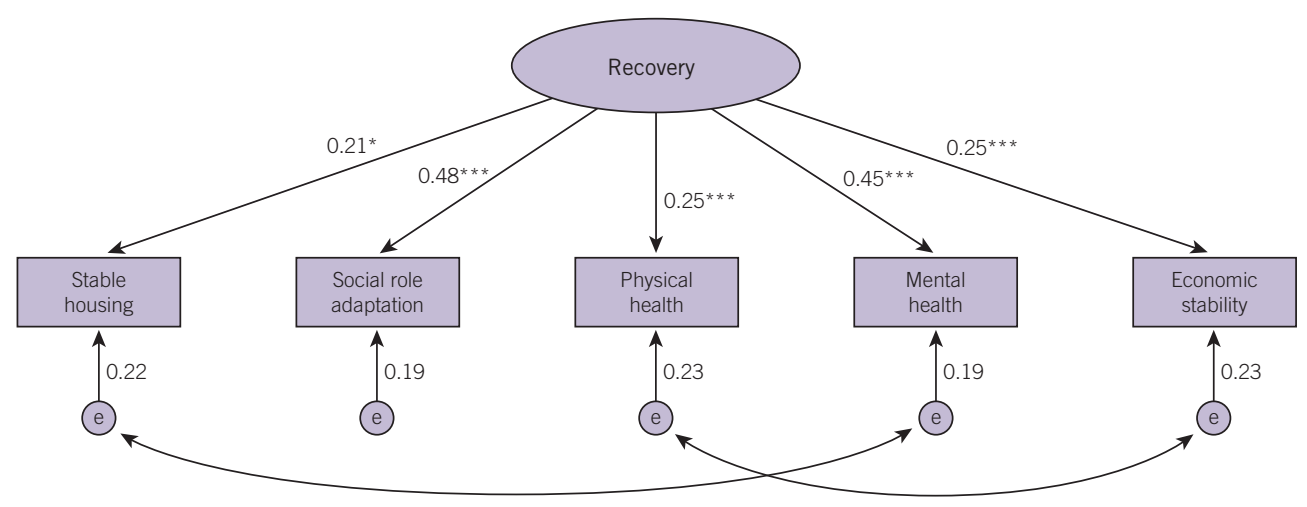

Tucker-Lewis Index 0.96; Comparative Fit Index 0.99; root mean square error of approximation 0.028; $\chi^{2} 4.98$; degrees of freedom 3. e=error.

$* P<.05$.

$* * P<.01$.

$* * * P<.001$. 


\section{RESULTS}

Latent variables, including the recovery measure, were developed as discussed in the Methods section; Figure 2 displays the factor loadings and fit of the model. Following standard convention used in SEM, observed variables are displayed in boxes and latent variables are shown in circles. The factor loadings presented in Figure 2 show standardized estimates that can be interpreted as correlation coefficients, although they are actually regression coefficients corresponding to the latent recovery variable. The factor loadings exhibit the strength in association between the observed variable and the latent variable. Among variables used to create the recovery measure, social role adaptation and mental health were most positively associated, with factor loadings of 0.48 and 0.45 , respectively $(P<.001)$. Physical health, economic stability, and stable housing were also positively correlated, but at about half the magnitude $(0.25,0.25,0.21$, respectively). Residual variance of the observed variables is shown to indicate the magnitude of variability in the error terms. Following the recommended model indices of $\mathrm{Hu}$ and Bentler, ${ }^{32}$ fit statistics for the recovery measure were good. The Tucker-Lewis Index (TLI) and Comparative Fit Index (CFI) should be close to 1 for a good fitting model; they were both excellent in this analysis, with the TLI 0.96 and the CFI 0.99. Good models also have a root mean square error of approximation (RMSEA) of $<0.07$; the RMSEA for the recovery factor was good at 0.028 . As a measure of face validity, a $t$ test was run to investigate the relation between our recovery construct and a subjective, self-assessed measure of recovery taken from the GCAFH survey. Participants who responded that their situation was better than or similar to what it had been before Hurricane Katrina had significantly greater recovery scores than participants who responded otherwise, $t(842)=-10.56 ; P<.001$.

In addition to the recovery measure, 2 additional latent factors were created for preexisting psychological strength and neighborhood characteristics. Figures 3 and 4 show the results of the CFA. In order of magnitude, psychological strength was positively associated with locus of control $(0.76 ; P<.001)$, selfefficacy $(0.37 ; P<.001)$ and religiosity $(0.15 ; P<.05)$. Locus of control was primarily driven by disagreement with the statements "People like me don't have a very good chance to be successful in life" and "Every time I try to get ahead, something or someone stops me" (factor loadings of 0.60 and 0.49 , respectively; $P<.001)$. A weaker but highly significant correlation existed between locus of control and agreement with the statement "I can handle most things that happen in my life" $(0.29$; $P<.001)$. The fit for the psychological strength model was excellent, with a TLI and CFI of 1.00 and RMSEA of 0.001 .

The neighborhood characteristics factor was based on 2 subfactors: social and physical disorder. The correlation between these 2 domains was 0.70 with $P<.001$. Social disorder was significantly correlated $(P<.001)$ with each of its variables; the highest correlation was with concern about being robbed (factor loading 0.79 ) and weakest with concern about their child being out at night (factor loading 0.66). Similarly, physical disorder was correlated with all of the variables, ranging from 0.74 for abandoned cars to $\mathbf{0 . 6 0}$ for graffiti on buildings. Estimates for the model fit ranged from very good, according to the TLI and CFI (0.93 and 0.95, respectively), to adequate, as indicated by the RMSEA of 0.073 .

\section{FIGURE 3}

\section{Factor loadings for the psychological measurement model.}

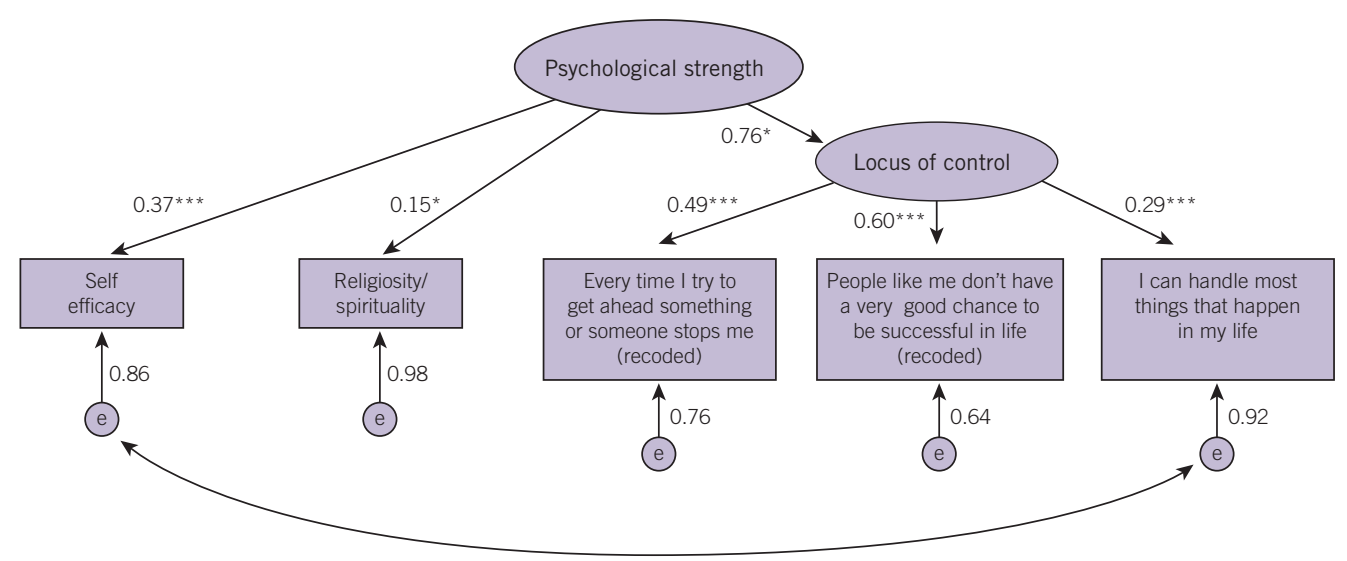

Tucker-Lewis Index 1.00; Comparative Fit Index 1.00; root mean square error of approximation 0.001; $\chi^{2} 2.58$; degrees of freedom 4. e=error

$* P<.05$.

$* * P<.01$.

$* * * P<.001$ 


\section{FIGURE 4}

\section{Factor loadings for the social/physical neighborhood measurement model.}

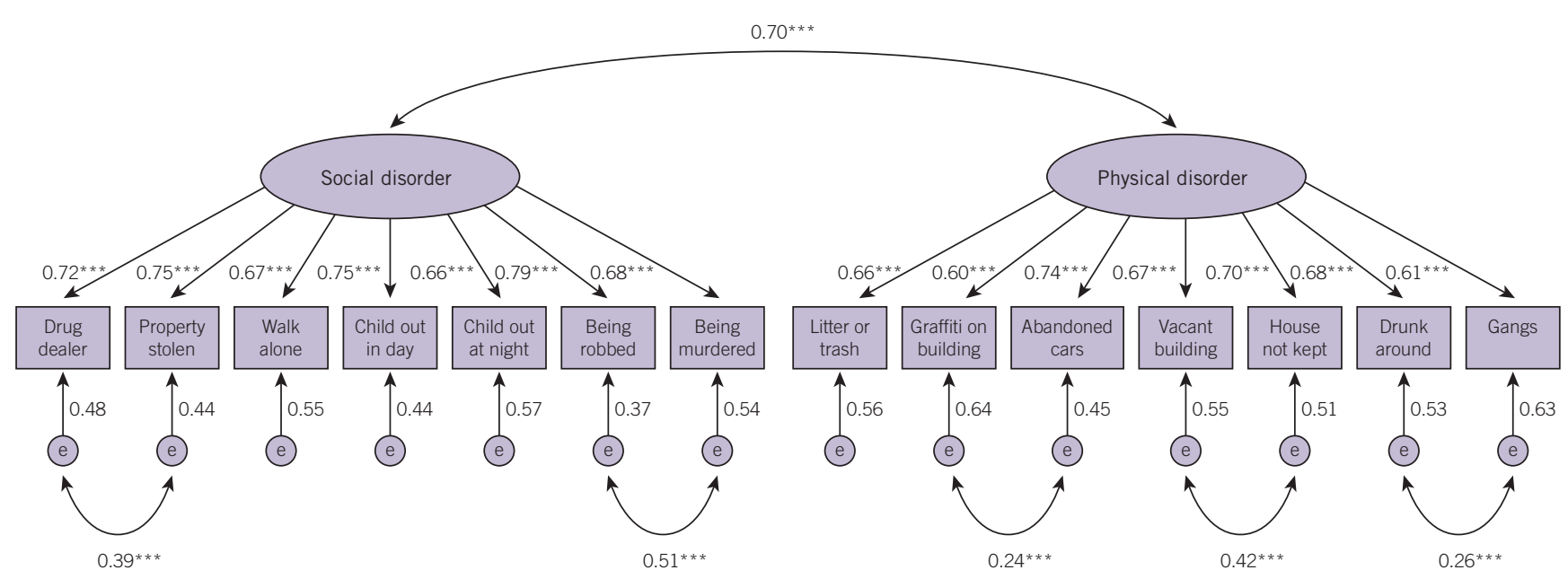

Tucker-Lewis Index 0.93; Comparative Fit Index 0.95; root mean square error of approximation 0.073; $\chi^{2} 387.84$; degrees of freedom 71 . e=error

$* P<.05$.

$* * P<.01$.

$* * * P<.001$

\section{TABLE 1}

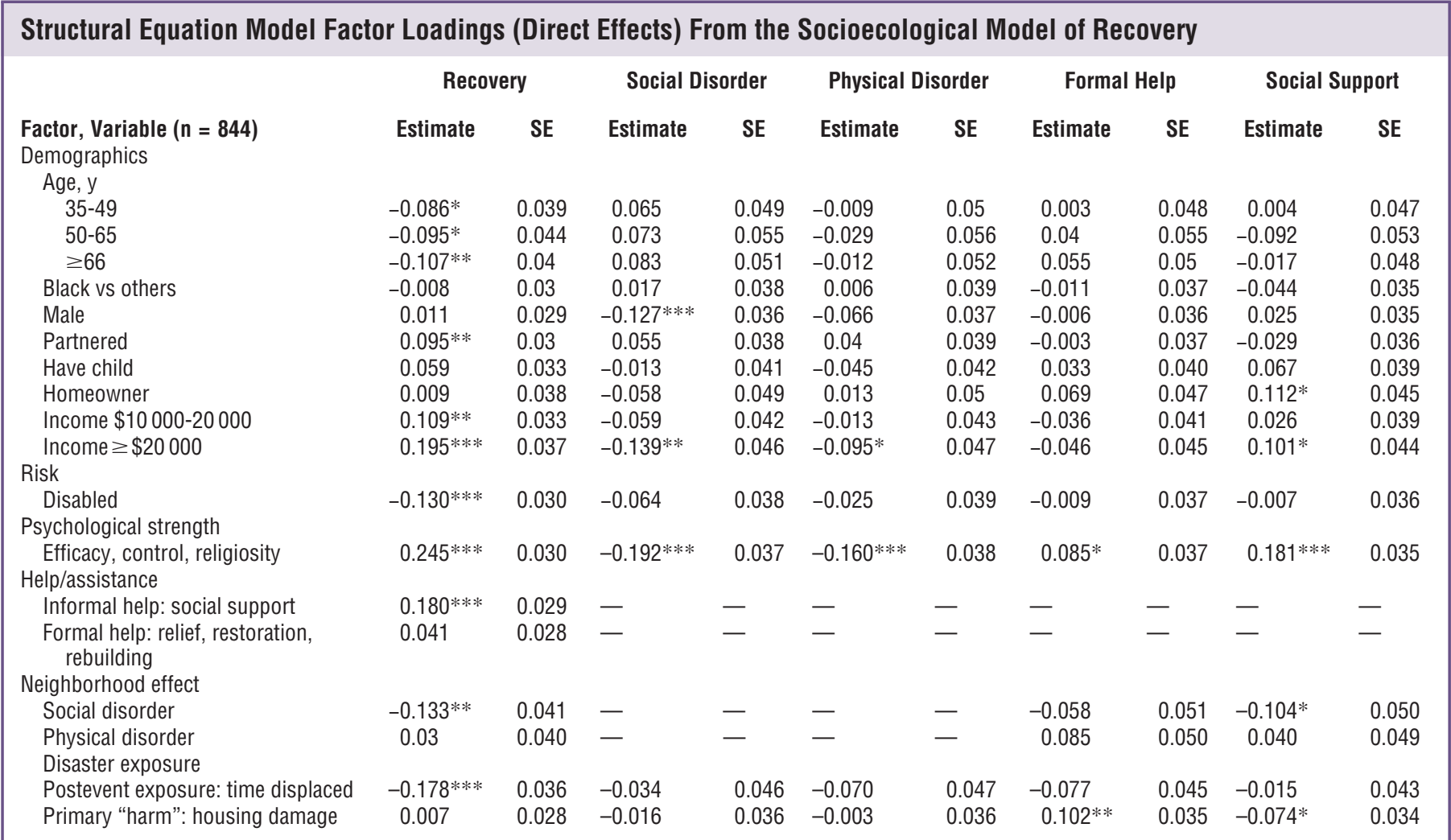

The columns represent outcomes. The Comparative Fit Index of the specified model was 0.99 and the root mean square error of approximation was 0.059 , which are within the recommended range for good model fit. The estimates reflect standardized coefficients.

$* P<.01$.

$* * P<.01$.

$* * * P<.001$ 
Results from the structural equation model showed excellent fit (CFI 0.99 and RMSEA 0.059). These indices validate the statistical fit of the model to infer that the theorized model matches the empirical data well. With the exception of race, all of the predisposing variables (listed on the left in Figure 1) had significant effects on recovery (Tables 1 and 2). Recovery, as measured by the recovery factor, increased by approximately 0.1 with each categorical increase in income (referenced against households with annual income $<\$ 10000$ ). There was an additional indirect benefit of 0.03 among people in the highest income group ( $>$ \$20 000) through social support and social disorder. Psychological strength had the strongest direct effect on recovery; every unit increase in psychological strength led to a $0.25-\mathrm{U}$ increase in recovery. Indirectly, psychological strength increased recovery an additional 0.06 via social support and social disorder. Living with a partner directly improved recovery by 0.1 , whereas indirect improvements were observed for homeownership at the time of the hurricane through social support and male gender via social disorder. Children living in the household did not have significant direct or indirect effects, but the total effect on recovery was positive $(0.07 ; P<.05)$.

Age and disability had negative direct effects on recovery. Compared to the youngest group, ages 18 to 34 years, people in older age groups had recovery scores approximately 0.1 lower. The upper middle-age group (50-65 years) had a small, negative, indirect effect as well; however, the effect was not significantly due to social support, formal help, social disorder, or physical disorder. Disability had the strongest negative effect on recovery, decreasing it by $0.13(P<.001)$.

Each of the 3 independent factors in the socioecological modeldisaster exposure/harms, neighborhood characteristics, and help—significantly affected recovery (Tables 1 and 2). Disaster exposure had a negative effect on recovery, decreasing it by $0.18(P<.001)$, with longer time displaced and indirectly by

\section{TABLE 2}

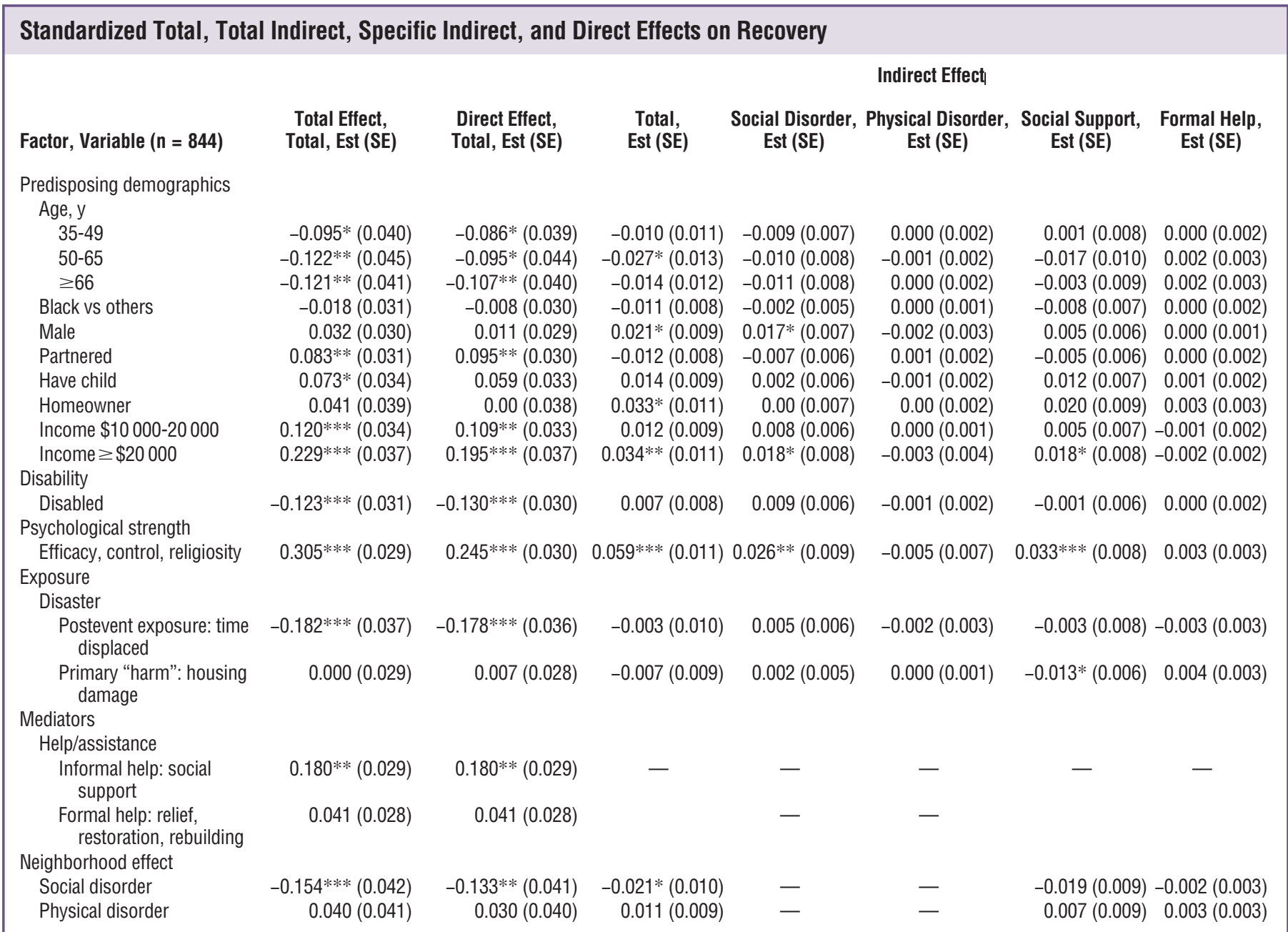

The Comparative Fit Index of the specified model was 0.99 and the root mean square error of approximation was 0.059 , which are within the recommended range for good model fit. The estimates (Est) reflect standardized coefficients.

$* P<.01$.

$* * P<.01$.

$* * * P<.001$ 
$0.01(P<.05)$ due to housing damage lowering social support. Neighborhood social disorder decreased recovery directly by 0.13 $(P<.01)$ and indirectly by $0.02(P<.05)$, primarily through reducing social support. Physical disorder had no significant effect on recovery. Informal help, measured by social support, significantly improved recovery by $0.18(P<.001)$, whereas formal help was not significant.

\section{COMIMENT}

Recovery has been studied traditionally as a unidimensional factor without taking into account the multiple systems that characterize change in an individual after a disaster. The present study combined 5 dimensions-housing stability, economic stability, good physical health, good mental health, and social role adaptability-into a single multidimensional factor that encompassed the foundations of recovery and investigated factors that may be involved in the pathway to recovery. Exposure to the disaster, neighborhood effects, and perceived and received help potentially affect an individual's recovery. Furthermore, the role of psychological strength and measures of risk such as disability can be moderators that influence a multitude of pathways. In short, the system of recovery is complex, with numerous factors that may require simultaneous examination. The socioecological model of recovery bridges these ideas into a single framework, and this study makes an unprecedented attempt at modeling these factors.

The method of analysis undertaken by this study has $2 \mathrm{com}$ ponents: developing a comprehensive operational measure that takes into account the 5 dimensions of recovery and a model that evaluates factors that influence this construct through multiple pathways and systems. As the results showed, the statistical fit of the measures developed exhibited excellent fit, which establishes inferences on the reliability and validity of the recovery measure we proposed. Furthermore, the fit of the structural equation model that incorporates the direct and indirect effects also showed good fit, adding to the finding that the socioecological model of recovery as a whole explains the empirical data well. These measures of model fit are meaningful because they provide an indication of whether the proposed model can appropriately account for theories applied in previous studies.

In addition to generating measures of model fit, the methodological framework provided a more robust method of analyzing the relation between factors, because the impact of a variable on recovery can be partitioned into its direct and indirect effects. This is important because significant indirect effect means that there are other mediating factors that channel the variability of a factor. Among sociodemographic factors that influenced recovery, we found that being of a younger age, having a higher household income, being partnered, and having a child had a significant and positive direct effect on recovery. The direct effect of homeownership on recovery was insignificant, but its indirect effect was significantly characterized by the mediating effect of social support, an informal mechanism of help. Psychological strength has both direct and indirect effects on recovery; its positive effect is mediated by both social support and social disorder to increase its total effect on recovery. The indirect effects of the mediating variables were also noteworthy. For example, the total effect of social disorder on recovery was created as a result of combinations of its direct effect and the indirect effect channeled via social support. It should be noted here that mediating factors such as neighborhood effects and help are external forces that can aid an individual's recovery; they have policy implications that can alleviate the effect of the disaster and expedite recovery.

\section{CONCLUSIONS}

Clearly, what we measure influences to what we attend. If the measure of recovery is the number of homes rebuilt in a community or the number of jobs restored, then construction and employment will be the primary foci of planners' attention. The present analysis presents a more nuanced recovery measure, and one that suggests that social processes such as the restoration of an individual's social support network, his or her capacity to recreate positive social interactions within the family, and even the resumption of a neighborhood's social order are critically important to an individual's recovery. Attending to these processes is far more complicated than resurrecting critical infrastructure, but they may be vitally important if public priorities include the recovery of individuals after a disaster.

Although this model has been developed to test disaster recovery at an individual level, it may also have value in estimating community-level recovery. Contrasting population levels of housing and economic security and physical and mental health before and after a disaster-available as secondary data from census and ongoing population surveys such as the Behavioral Risk Factor Survey and the National Health Interview Studymay offer clues to a community's long-term recovery. With the clues come many limitations: it may take years to collect the necessary population data, the smallest geographic units for analysis may be county-level units, and there may be significant selection biases of the population surveyed postdisaster. This postdisaster population may represent one with greater advantages or access to resources, particularly if the less advantaged have been permanently displaced. Furthermore, 1 central element of this proposed recovery measure, social role adaptation, is not routinely collected in population surveys.

Despite such limitations, validated measures of individual recovery can be used to test community-level proxy measures. One of the long-standing weaknesses of the social vulnerability literature ${ }^{1}$ has been its inability to validate the relation of its objectively drawn measures to clear outcomes. This subjective recovery measure could provide such a test. Once validated, these community-level proxy measures could be used to explore recovery after an event and to predict recovery before the occurrence of an event, when mitigation and preparedness efforts could be deployed to their greatest effect. 
The limitations of this study suggest a number of future avenues for research. Because this analysis was conducted at only a cross-sectional level, a longitudinal approach that measures the rate of change in an individual's recovery trajectory over time could be an important step in understanding the nature of recovery after disasters. Moreover, the external validity or generalizability of this model must be empirically tested using other populations and other disaster settings to truly make implications of understanding behaviors and systems that affect recovery. The component items of the recovery measure should be broadened, particularly within the measures of formal help and social role adaptation. Finally, a true test of a socioecological framework of recovery would establish the empirical relation between the process of recovery and recovery as an outcome: that specific actions and policies can be demonstrated to accelerate individuals' capacity to achieve stable housing and economic circumstances, to restore their optimal mental and physical well-being, and to reestablish themselves as productive social actors within their families and their communities.

Author Affiliations: All of the authors are with the National Center for Disaster Preparedness, Columbia University.

Correspondence: Address correspondence and reprint requests to Dr David Abramson, National Center for Disaster Preparedness, Columbia University, 215 W 125th St, Suite 303, New York, NY 10027 (e-mail: dma3@columbia .edu).

Received for publication: Received for publication July 16, 2010; accepted August 2, 2010 .

Authors' Disclosures: The authors report no conflicts of interest.

\section{REFERENCES}

1. Cutter SL, Boruff BJ, Shirley WL. Social vulnerability to environmental hazards. Soc Sci Q. 2003;84:242-261.

2. Green B, Solomon S. The mental health impact of natural and technological disasters. In: Hobfoll JFaS, ed. Traumatic Stress: From Theory to Practice. New York: Plenum; 1995:163-180.

3. Benight CC, Bandura A. Social cognitive theory of posttraumatic recovery: the role of perceived self-efficacy. Behav Res Ther. 2004;42(10):1129. 1148.

4. Norris FH, Stevens SP, Pfefferbaum B, Wyche KF, Pfefferbaum RL. Community resilience as a metaphor, theory, set of capacities, and strategy for disaster readiness. Am J Community Psychol. 2008;41(1-2):127-150.

5. Bates FL, Pelanda C. An ecological approach to disasters. In: Dynes RR, Tierney KJ, eds. Disasters, Collective Behavior, and Social Organization. Newark: University of Delaware Press; 1993:145-159.

6. Edwards MLK. An interdisciplinary perspective on disasters and stress: the promise of an ecological framework. Sociol Forum. 1998;13:115-132.

7. Kothari A, Edwards N, Yanicki S, et al. Socioecological models: strengthening intervention research in tobacco control. Drogues Sante Societe. 2007;6:iii5-iii6.

8. Tatsuki S. Long-term life recovery processes among survivors of the 1995 Kobe earthquake: 1999, 2001, 2003, and 2005 Life Recovery Social Survey results. J Disaster Res. 2007;2:484-501.
9. Koshiyama K. Analysis of sense of recovery of residents living in disaster recovery public housing. J Inst Soc Safety Sci. 2003;3:237-244.

10. Tatsuki S, Hayashi H. Family system adjustment and adaptive reconstruction of social reality among the 1995 earthquake survivors. Int J Jpn Sociol. 2000;9:81-110.

11. Bratt RG. Housing and family well-being. Housing Stud. 2002;17:13-26.

12. Peacock WG, Dash N, Zhang Y. Sheltering and housing recovery following disaster. In: Rodriguez H, Quarantelli EL, Dynes RR, eds. Handbook of Disaster Research. New York: Springer; 2006:258-274.

13. Fothergill A, Peek LA. Poverty and disasters in the United States: a review of recent sociological findings. Nat Hazards. 2004;32:89-110.

14. Enarson E, Morrow BH. The Gendered Terrain of Disaster: Through Women's Eyes. Westport, CT: Praeger; 1998.

15. Morrow BH. Stretching the bonds: the families of Andrew. In: Peacock WG, Morrow BH, Gladwin H, eds. Hurricane Andrew: Ethnicity, Gender and the Sociology of Disasters. London: Routledge; 1997:141-170.

16. National Research Council. Facing Hazards and Disasters: Understanding Human Dimensions. Washington, DC: National Academies Press; 2006.

17. Smith GP, Wenger D. Sustainable disaster recovery: operationalizing an existing agenda. In: Rodriguez H, Quarantelli EL, Dynes RR, eds. Handbook of Disaster Research. New York: Springer; 2006:234-257.

18. Heller T. The effects of involuntary residential relocation: a review. Am J Community Psychol. 1982;10(4):471-492.

19. Reich JW. Three psychological principles of resilience in natural disasters. Disaster Prev Manage. 2006;15:793-798.

20. Silove D, Steel Z. Understanding community psychosocial needs after disasters: implications for mental health services. J Postgrad Med. 2006; 52(2):121-125.

21. Chamlee-Wright ER. The long road back: signal noise in the postKatrina context. Independent Rev. 2007;12:235-259.

22. Chamlee-Wright ER, Daniel M. Disastrous Uncertainty: How Government Disaster Policy Undermines Community Rebound. Mercatus Policy Series, Policy Comment No. 9. Arlington, VA: Mercatus Center at George Mason University; 2007:29.

23. Abramson D, Stehling-Ariza T, Garfield R, Redlener I. Prevalence and predictors of mental health distress post-Katrina: findings from the Gulf Coast Child and Family Health Study. Disaster Med Public Health Prep. 2008;2(2):77-86.

24. Lee SY. Structural Equation Modeling: A Bayesian Approach. New York: Wiley; 2007.

25. Ware JE Jr, Kosinski M, Keller SD. A 12-Item Short-Form Health Survey: construction of scales and preliminary tests of reliability and validity. Med Care. 1996;34(3):220-233.

26. Ware JE Jr. Improvements in short-form measures of health status: introduction to a series. J Clin Epidemiol. 2008;61(1):1-5.

27. Bollen KA. Structural Equations with Latent Variables. New York: John Wiley \& Sons; 1989.

28. Schwarzer R, Jerusalem S. Generalized self-efficacy scale. In: Weinman J, Wright S, Johnston M, eds. Measures in Health Psychology: A User's Portfolio. Causal and Control Beliefs. Windsor, UK: NFER-NELSON; 1995: 35-37.

29. Allen MJ, Yen WM. Introduction to Measurement Theory. Long Grove, IL: Waveland Press; 2002.

30. Cook TD, Campbell DT. Quasi-Experimentation: Design and Analysis Issues in Field Settings. Boston: Houghton Mifflin; 1979.

31. Bagozzi RP, Yi Y, Phillips LW. Assessing construct validity in organizational research. Adm Sci Q. 1991;36:421-458.

32. Hu LT, Bentler PM. Cutoff criteria for fit indexes in covariance structure analysis: conventional criteria versus new alternatives. Struct Equ Modeling. 1999;6:1-55. 